\title{
iSGAT: Gestión colaborativa de tesauros en Internet
}

\author{
Juan Antonio Pastor Sánchez \\ Francisco Javier Martínez Méndez \\ Grupo de Tecnologías de la Información \\ Universidad de Murcia
}

\subsection{Resumen}

Se presenta el diseño de un sistema integrado de gestión de tesauros distribuido y cooperativo denominado iSGAT basado en una arquitectura de base de datos relacional interoperable en la World Wide Web. El sistema permitirá la asignación de descriptores a direcciones de Internet, configurando así un modelo de recuperación de información para la web basado en descriptores. De esta forma, diferentes grupos de trabajo podrán desarrollar unos repositorios de conocimiento previamente indizados, que favorecerán la difusión de los documentos de mayor interés en sus áreas de trabajo. Esto convierte a iSGAT en una herramienta ideal para comunidades virtuales de usuarios dentro del ámbito de la investigación científica y tecnológica.

Palabras clave: Recuperación de información. Indización. Tesauros. World Wide Web. Aplicaciones de trabajo en grupo. Sistemas integrados de gestión de tesauros. Gestión del Conocimiento.

\subsection{Abstract}

The design of a distributed, integrated and collaborative thesauri management system for the World Wide Web, named iSGAT, is presented. The system is based on a relational database architecture. It allows the online description of web resources; so that several groups can develop cooperatively knowledge repositories, properly indexed, that will support the dissemination of interesting documents in their areas of work. This turns iSGAT in an ideal tool for virtual communities in the field of scientific and technological research.

Keywords: Information Retrieval. Indexing. Thesuari. Web. Groupware. Integrated Thesauri Management Systems. Knowledge Management. 


\section{Introducción}

La elaboración de tesauros ha sido durante mucho tiempo una tarea compleja. Dicha complejidad se ha debido fundamentalmente a la necesidad de coordinar distintos grupos de trabajo y al uso de múltiples fuentes de información en la elaboración de la terminología y su posterior estructuración. La utilización de aplicaciones informáticas especializadas para la construcción de estos tesauros proporciona una mayor rapidez en el proceso de creación de estos lenguajes documentales, fomentando así su uso en los centros e instituciones documentales. Asimismo, la gestión informática permite un control más eficaz de los términos, aumentando el control de su consistencia. Por otro lado, la gestión informática favorece la generación automática de distintos tipos de presentaciones e índices. Estas ventajas han propiciado el desarrollo de un conjunto de aplicaciones informáticas con este propósito. Una reciente y completa revisión del software utilizado para la gestión de tesauros, ha sido realizada por Moya Martínez y Gil Leiva en un trabajo publicado en la revista Ciencias de la Información (2001). Este artículo evaluaba las características de cinco paquetes informáticos: BEAT, MultiTes, Stride, TCS-Y2K y SGAT, este último desarrollado por desarrollado por el técnico de información y profesor de la Universidad de Murcia, Juan Antonio Pastor Sánchez, coautor de este trabajo. Las conclusiones principales de este trabajo destacan una adecuada gestión de las relaciones y de los formatos de visualización, no pudiéndose decir lo mismo de los formatos de impresión de los tesauros y de la gestión de las notas explicativas; también se valoraban positivamente las capacidades de exportación de ficheros a otros sistemas.

En Internet es cada vez más frecuente encontrar tesauros en línea que generalmente permiten la consulta de sus términos y sus relaciones. Una amplia selección de estos tesauros en línea la encontramos en el trabajo de Alejandro de la Cueva (2000) y en la página web de Eva Méndez Rodríguez (2002b), dedicada al soporte para la construcción de tesauros en Internet, donde recoge algunos de tesauros analizados en el trabajo anterior y presenta varias recopilaciones de tesauros en línea, junto a un conjunto de planteamientos teóricos y metodológicos sobre la construcción de tesauros. La mayoría de los tesauros en línea que podemos encontrar en la web, a pesar de su variedad temática, se agrupan básicamente en dos únicas categorías: presentaciones sistemáticas o alfabéticas y diccionarios terminológicos. Un ejemplo del primer grupo es el Tesauro de Redes de Ordenadores (Martínez Méndez y García Gómez, 2003) (desarrollado con el sistema SGAT), del cual mostramos un fragmento de su presentación sistemática. Verdaderamente, este tipo de tesauros sólo hace uso de la web para difundir sus contenidos y aumentar así su utilidad.

El segundo grupo más abundante de tesauros en línea son los diccionarios de términos, más o menos elaborados y que aprovechan la interactividad de la web para facilitar las consultas a su contenido. De este segundo grupo de tesauros en

Scire. $9: 2$ (jul.-dic. 2003) 85-98. 
línea, proponemos como ejemplo la web Hypertext Webster Gateway (http: //www.bennetyee.org/ http_webster.cgi?), que proporciona una interface de consulta a algunos de los principales diccionarios en línea de Internet. $\mathrm{Su}$ uso es muy simple, basta con insertar el término a buscar en el recuadro de texto correspondiente, y el sistema realiza la búsqueda pertinente en varios diccionarios al mismo tiempo. La siguiente imagen muestra el resultado de una búsqueda de la entrada "Information Science". Este sistema, además de presentar al usuario una definición del término solicitado, aprovecha las características hipertextuales de la web para proporcionar acceso a las definiciones de los términos empleados en ella.

Si al uso de la web como medio de presentación del tesauro o como vínculo interactivo para su consulta, le añadiéramos la posibilidad de realizar la construcción del tesauro en línea por parte de un grupo de trabajo en red, las prestaciones de estos sistemas aumentarían enormemente, especialmente en los procesos de implementación y mantenimiento, y, lógicamente, en las operaciones de recuperación de información).

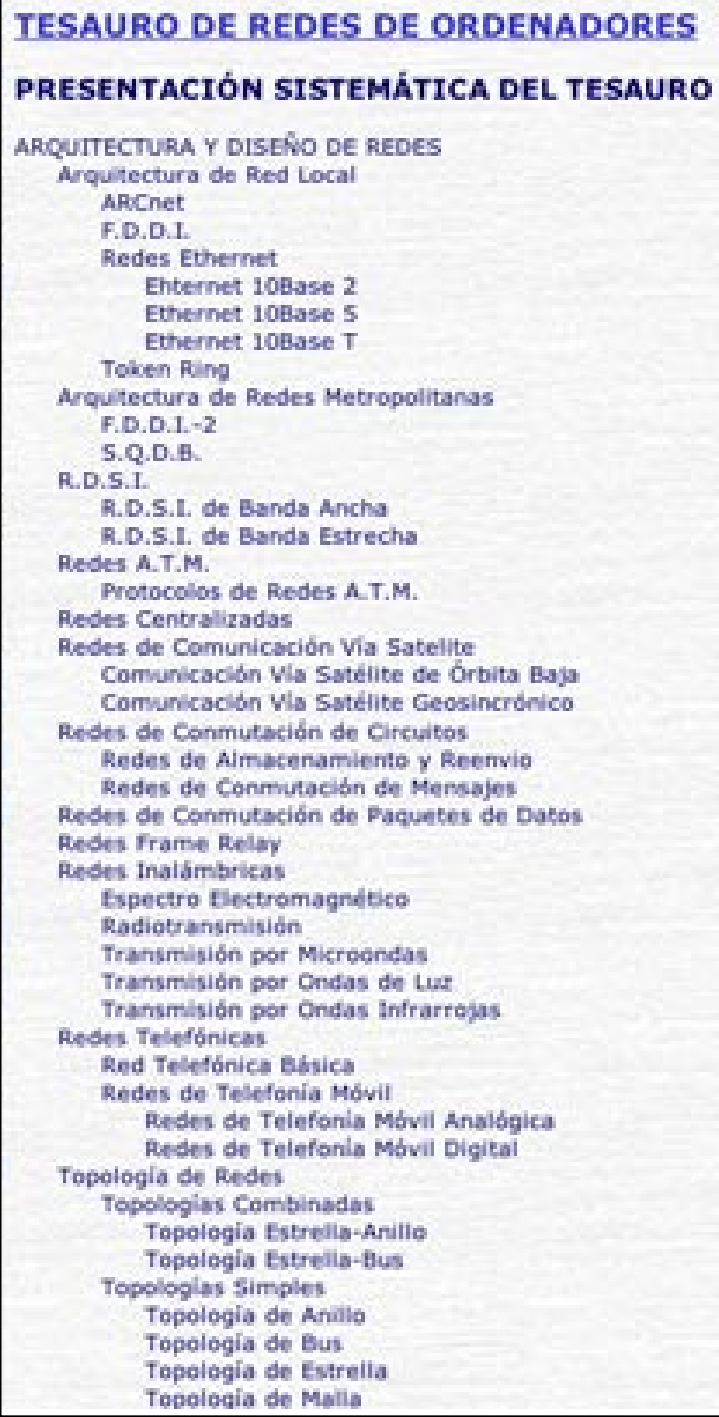

Fig. 1. Sección Tesauro de Redes de Ordenadores. 


\title{
Hypertext Webster Gateway: "Information Science"
}

\author{
From WordNet (r) 1.7 (wn)
}

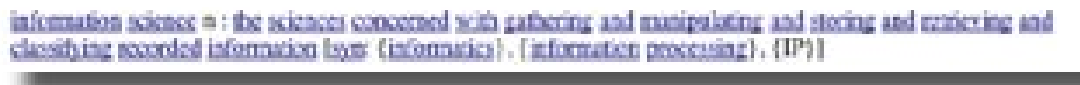

Fig. 2. Sección de la web Hypertext Webster Gateway.

Internet en general $-\mathrm{y}$ la web en particular - constituyen unos espacios de intercambio y colaboración científica muy adecuados para la implantación de sistemas gestores de tesauros en línea, ya que:

- Internet permite establecer grupos de trabajo, incluso muy alejados en espacio y banda horaria, que pueden colaborar en la elaboración de un tesauro. El uso de bases de datos relacionales a través de una interfaz web,y del correo electrónico permitirían establecer una dinámica de trabajo alrededor de una serie de flujos de trabajo para la creación y mantenimiento de términos y relaciones del tesauro.

- La filosofía cliente-servidor subyacente en todas las aplicaciones de la red Internet (y muy particularmente en la web) permitirá construir en común un corpus léxico documental, íntegramente adaptable a las características particulares de cada usuario, por medio del desarrollo de aplicaciones cliente específicas para diversos propósitos.

- De forma casi consutancial con la propia naturaleza de la web como sistema de información, se pueden extender las capacidades del tesauro gestionado, convirtiéndolo en un instrumento muy válido para la recuperación de información.

En definitiva, con el desarrollo de iSGAT, se pretende construir un sistema que permita el desarrollo de tesauros en línea dentro de la red Internet, aprovechando al máximo las prestaciones de sistema cliente/servidor de la tecnología web, y convertirlo en un medio para un óptima recuperación de información dentro de una colección de documentos previamente indizados.

\section{Objetivo y fases del proyecto}

Una herramienta informática de la naturaleza de iSGAT permitirá construir tesauros en línea de forma sencilla y altamente interactiva. Además, posibilitará implantar un control de usuarios para la identificación de los mismos, un sistema de validación de los contenidos y un sistema de consulta del propio tesauro. De forma adicional, este tesauro podría emplearse para la indización de un conjunto específico de documentos (o repositorio de documentos), e iSGAT realizaría de

Scire. $9: 2$ (jul.-dic. 2003) 85-98. 
forma suplementaria la labor de motor de búsqueda, aprovechándose de la indización previa. Gracias a la interactividad y versatilidad de la web, estos recursos pueden formar parte de la Intranet de una organización o pueden ser depositados directamente en la propia web. Para el logro de estos objetivos, resulta necesario establecer una serie de fases de desarrollo que permitan pasar de la concepción inicial del sistema iSGAT a su implementación definitiva:

A. Desarrollo conceptual del sistema: En esta fase se han de identificar las funciones y los elementos de cada uno de los módulos y entidades que conformarán posteriormente el sistema.

B. Implementación en entorno Web: Tras realizar la descripción conceptual, hay que proceder a desarrollar los diferentes módulos para su funcionamiento a través de Internet. Será en este momento cuando se decida qué herramientas de trabajo emplear: lenguajes de programación, gestor de bases de datos, interfaz de usuario, etc.

C. Conversión de tesauros especializados: Una vez desarrollado un prototipo del sistema, se introducirán algunos tesauros especializados y se utilizarán para la indización de recursos de Internet e Intranet, con el fin de verificar el funcionamiento de los módulos implementados y de valorar las prestaciones del trabajo en grupo.

D. Apertura del sistema a cualquier usuario, tras las verificaciones efectuadas sobre el diseño original, fruto de la fase anterior.

De este conjunto de fases destaca, en virtud de su complejidad procedimental, la dedicada a la implementación de la aplicación informática, que va a seguir la siguiente serie de pasos:

1. Definición clara y concisa de las operaciones de gestión de tesauros sistemáticos monolingües y agrupamiento de estas operaciones en perfiles de usuarios. Esto permitirá el establecimiento de un entorno de trabajo en grupo y la determinación de una serie de flujos de trabajo, así como el control de las fuentes de información utilizadas para la elaboración de la terminología y su estructuración.

2. Descripción de las funciones propias de gestión y consulta del tesauro en un sistema informático distribuido, basado en una interfaz común y en una arquitectura cliente/servidor. En este punto resulta esencial la especificación de la forma en la que se crean, validan y eliminan los descriptores, no descriptores y los campos semánticos del tesauro, así como la dinámica de creación de relaciones y los distintos modos de consulta y navegación.

3. Desarrollo de un sistema de indización de documentos en Internet en el cual se asocien descriptores y documentos. A través de un lenguaje 
de interrogación se podrán recuperar documentos con base en los descriptores del sistema, reutilizando los resultados para obtener una mayor eficacia en la recuperación de información.

\section{Entorno de Trabajo en Grupo para la gestión de tesauros con iSGAT}

Dentro de las tareas propias de un entorno de trabajo en grupo, se establece una dinámica de reparto de tareas basada en un sencillo esquema de flujo de trabajo. Es posible definir una serie de funciones que pueden realizar los usuarios dentro del proceso de gestión de un tesauro:

- Crear tesauro: El propósito de esta función es la de crear las tablas relacionales que sirvan de base para el tesauro.

- Crear usuarios: El administrador o el responsable del mantenimiento del tesauro podrá crear nuevos usuarios asociados, especificando ciertos datos de identificación (nombre, palabra clave y correo electrónico) y definiendo las funciones asignadas dentro de la dinámica de flujo de trabajo.

- Gestionar y validar términos, relaciones o indización: El usuario que tiene asignada alguna de estas funciones puede crear nuevos términos, relaciones o emparejamientos entre descriptores y recursos Internet; o bien validar los que han propuesto los usuarios que tienen asignadas las funciones correspondientes para proponer términos, relaciones o indización.

- Proponer términos, relaciones o indización: Es necesaria la separación entre el gestor de términos, relaciones o indización, y los usuarios que proponen la creación, modificación o supresión de dichos elementos. Al realizar una propuesta, dicho elemento debe validarse por parte del gestor correspondiente para que los cambios se incorporen al tesauro. La definición de los términos permitirá la inclusión de notas explicativas y definición de los mismos, si se considera necesario.

- Control de fuentes: Esta función permite registrar las fuentes de información que se han utilizado para la obtención de la terminología del tesauro. Así, posteriormente se pueden asociar dichas fuentes a los diferentes términos y realizar revisiones o actualizaciones.

- Consultar indización: Mediante una serie de formularios de búsqueda, integrados con una interfaz de navegación del tesauro, los usuarios autorizados pueden recuperar los términos descriptores y los documentos asociados.

- Consultar tesauro: El usuario que tenga asignada está función podrá consultar el tesauro a través de la presentación alfabética o de la presentación sistemática. La consulta del tesauro lleva implícita la posibilidad de poder realizar búsquedas por descriptores. 
Según el número de funciones que tengan asignadas los distintos usuarios de iSGAT se pueden definir los siguientes perfiles de usuario, que interactuarán según el esquema de flujo de trabajo recogido en la siguiente ilustración:

- Administrador: Es el responsable de la creación de las tablas relacionales necesarias para la creación del tesauro y a la inclusión de nuevos usuarios en el sistema. Aunque también está habilitado para crear nuevos usuarios, como norma general sólo deberá crear a un usuario con dicho perfil.

- Gerente: Es el responsable de la gestión de contenidos e indización de un tesauro en iSGAT. También puede crear usuarios, a los que puede asignarles diferentes perfiles. Esta función también está disponible por los administradores aunque se recomienda que no la lleven a cabo.

- Editor: Este usuario tiene las mismas funciones que el gerente. Sin embargo, no le será posible crear nuevos usuarios. Sus funciones están orientadas únicamente a la gestión conceptual del tesauro.

- Autor: Como autor se entiende al usuario que puede proponer términos, relaciones e indizaciones; esto es, a los gestores del contenido. Los autores tienen asignado por defecto un editor que será el responsable de validar sus propuestas y que asume el papel de supervisor de la integridad del sistema.

- Lector: Los usuarios con este perfil podrán consultar el tesauro, así como realizar búsquedas en la tabla de indización. En último término, si el tesauro se implementa como sistema de indización y consulta de un repositorio de documentos, les será posible recuperar información.

- Anónimo: En realidad no se trata de un perfil, sino de un usuario especial que accede al sistema sin necesidad de un identificador de usuario ni de una clave. Por regla general el usuario anónimo tendrá asignadas solamente las funciones de consulta del tesauro y de la indización.

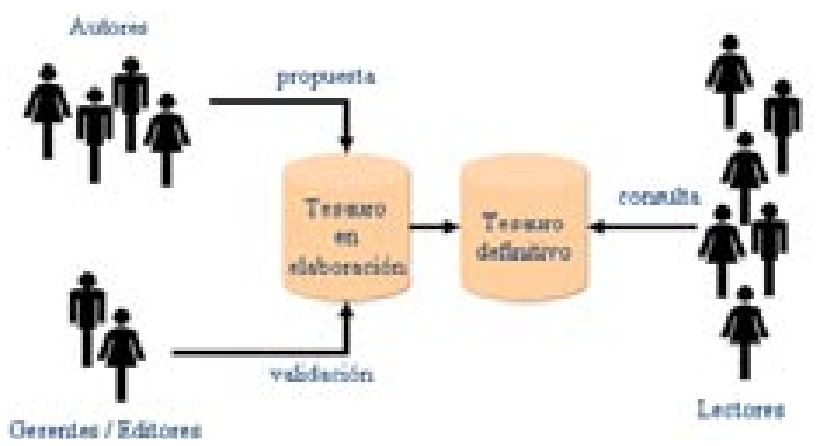

Fig. 3. Esquema del flujo de trabajo en el sistema iSGAT.

Scire. $9: 2$ (jul.-dic. 2003) 85-98. 


\begin{tabular}{|c|c|c|c|c|c|c|}
\hline & 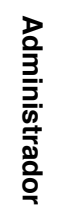 & 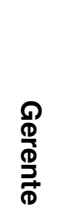 & $\frac{\text { m }}{\text { 음 }}$ & 胥 & 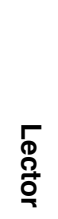 & 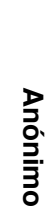 \\
\hline Crear tesauros & SI & NO & NO & NO & NO & $\mathrm{NO}$ \\
\hline Crear usuarios & SI & SI & NO & NO & NO & NO \\
\hline Gestionar y validar términos & NO & SI & $\mathrm{SI}$ & NO & NO & $\mathrm{NO}$ \\
\hline Gestionar y validad Relaciones & NO & SI & SI & NO & NO & NO \\
\hline Gestionar y validar Indización & NO & SI & $\mathrm{SI}$ & NO & NO & NO \\
\hline Proponer términos & NO & SI & SI & SI & NO & NO \\
\hline Proponer relaciones & NO & SI & $\mathrm{SI}$ & SI & NO & NO \\
\hline Proponer indización & NO & SI & $\mathrm{SI}$ & SI & NO & NO \\
\hline Control de fuentes & NO & SI & SI & $\mathrm{SI}$ & NO & NO \\
\hline Consultar indización & SI & SI & SI & SI & SI & SI \\
\hline Consultar tesauro & SI & SI & SI & SI & SI & SI \\
\hline Consultar repositorio & SI & SI & SI & SI & SI & SI \\
\hline
\end{tabular}

Tabla I. Perfiles de usuario y funciones dentro del sistema de trabajo en grupo de iSGAT. Fuente: elaboración propia.

\section{Funciones de gestión de tesauros}

En este apartado se van a presentar las funciones que iSGAT dispone para la gestión integral de los tesauros. Un tesauro precisa, para su correcta construcción por parte del grupo de trabajo encargado, de la definición previa de una serie de parámetros: el tipo de términos a manejar, el idioma o idiomas del tesauro, la naturaleza de las relaciones a establecer y su notación, las restricciones de tipo semánticos que pudieran establecerse y las presentaciones del contenido del tesauro. Para ello, en iSGAT se definirá el siguiente conjunto de operaciones.

- Tipología de términos: dentro del tesauro se van a distinguir dos tipos de términos, descriptores y no descriptores. Pueden utilizarse tanto unitérminos como términos compuestos. Ambos tipos de términos podrán incorporar una nota explicativa de su significado y/o función en el sistema 
de indización. También van a definirse los campos semánticos del tesauro, esto es, las grandes áreas sobre las que se va a estructurar el tesauro.

- Definición del idioma: iSGAT es un sistema orientado a la creación y gestión de tesauros a través de la web, por lo que cabe la posibilidad de que algún grupo de trabajo desee elaborar un tesauro multilingüe. Esta función, permite la gestión de hasta seis idiomas. Para cada idioma se deberá definir un usuario tipo Gerente (que puede ser el mismo en alguna ocasión).

- Naturaleza de las relaciones y notación: El sistema iSGAT lleva integradas las normas y recomendaciones recogidas en la norma ISO 2788 para tesauros monolingües y su traducción española UNE 50-106-90, y en la norma ISO 5964 para tesauros multilingües. A la hora de la creación del tesauro, y tras la indicación del número de idiomas del mismo, se elegirán las relaciones jerárquicas, asociativas y de sinonimia presentes en el mismo y se indicará cuál será el símbolo que las denota.

- Establecimiento de restricciones: En el momento de crear el tesauro resulta necesario establecer una serie de restricciones a los usuarios autores. Estas restricciones están relacionadas con la posibilidad de identificar distintos campos semánticos dentro de un tesauro y, en su caso, con el establecimiento de relaciones de tipo asociativo entre descriptores de distintos campos. Asimismo, iSGAT exige la adscripción de cada término a un campo semántico, con el fin de mantener homogénea la estructura semántica del tesauro. También deben incluirse restricciones relacionadas con la escritura de los términos del tesauro, que debe llevarse a cabo de forma unívoca - cual una clave primaria - ; y, si llegara a ser necesario, repetir la entrada de un término en el tesauro, éste debería ir acompañado de una nota. Por último, será necesario mantener una estructura coherente en la definición de las relaciones jerárquicas, evitando incongruencias semánticas.

La importancia que posee mantener la integridad y la coherencia del tesauro, pone de manifiesto la necesidad de desarrollar, dentro del sistema iSGAT, un módulo de verificación del cumplimiento de estas restricciones, que sólo podrá ser modificado por el usuario gerente, de manera que los autores se encontrarán trabajando dentro de una disciplina de grupo que garantizará la integridad de la indización. Una vez los gerentes y editores hayan realizado este conjunto de tares, corresponde a los autores la inserción de los componentes del tesauro, es decir, de sus términos y relaciones. Al mismo tiempo que van dotando de contenido al tesauro, van realizando alguna de las siguientes funciones:

- Inserción del término; y de sus notas explicativas, si procede.

- Adscripción de término descriptor o no descriptor a un campo semántico.

- Establecimiento de la equivalencia interlingüística (tesauros multilingües).

Scire. $9: 2$ (jul.-dic. 2003) 85-98. 
- Establecimiento de la equivalencia intralingüística (relación de sinonimia en tesauros monolingües).

- Establecimiento de relaciones de jerarquía.

- Establecimiento de relaciones asociativas.

Aunque es deseable que los autores (debidamente supervisados por los editores) no cometan fallos que afecten a la integridad y a la coherencia de la semántica del tesauro, el módulo de verificación de las restricciones de integridad actuará como garante de una adecuada edición de contenidos.

\section{Aplicación a la indización y recuperación en Internet}

Rodríguez Maniega recoge en su sitio web dedicado a los tesauros diferentes empleos de los mismos en Internet, siendo la recuperación de información el último de ellos. La autora muestra un ejemplo de aplicación de un tesauro en línea - más concretamente el Tesauro sobre el Síndrome de Inmunodeficiencia Adquirida elaborado por la iniciativa canadiense para el intercambio de información sobre esta enfermedad - como instrumento para mejorar la recuperación de información en bases de datos en línea (Rodríguez Maniega, 2000).

Tal como se ha comentado en la introducción de este trabajo, ésta es precisamente una de las ventajas adicionales del desarrollo de repositorios de conocimiento con iSGAT, ya que va a permitir a bibliotecas, centros de documentación, archivos o equipos de trabajos multidisciplinares recopilar recursos en la red que van a ser posteriormente indizados dentro del mismo entorno de trabajo, con el fin de facilitar a los usuarios lectores o invitados una recuperación de información óptima y eficaz sobre un campo determinado del conocimiento. iSGAT va a permitir cuatro modalidades de recuperación de información: a) navegación a través de la presentación alfabética o sistemática del tesauro; b) búsquedas por término exacto o aproximado, c) búsquedas sobre la metainformación de los recursos o sobre el conjunto de términos descriptores y no-descriptores que conformarán el tesauro: y c) al recuperar un documento se visualizarán los descriptores con los que ha sido indizado, lo que permitirá la realización de búsquedas posteriores de otros conjuntos de documentos terminológicamente relacionados.

Los repositorios de documentos gestionados por iSGAT han de tener importantes ventajas frente a los tradicionales directorios de recursos en la web. Los documentos van a ser descritos e indizados con una mayor exhaustividad y precisión que en un clásico directorio web. Al estar las operaciones de recuperación de información auxiliadas de un lenguaje documental construido por expertos profesionales e investigadores, mejora sustancialmente la efectividad de las búsquedas, pues el conocimiento de la estructura y el contenido del tesauro favorecerá la correcta elaboración de las consultas. Finalmente, la creación de repositorios

Scire. $9: 2$ (jul.-dic. 2003) 85-98. 


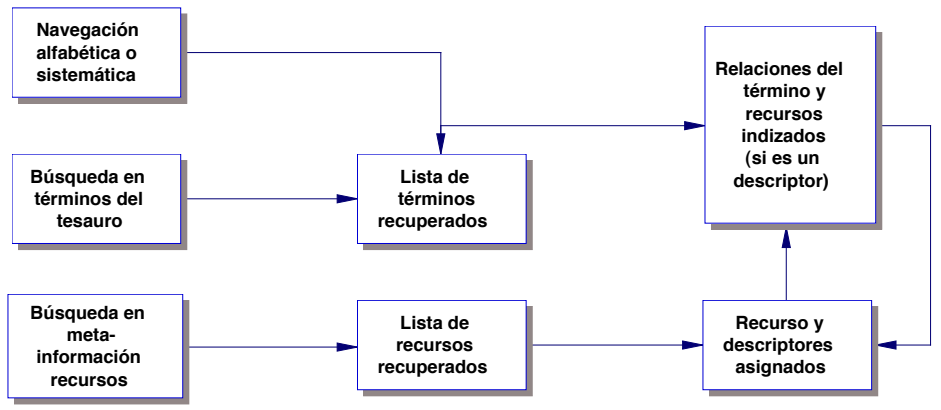

Fig. 4: Recuperación de información con iSGAT.

específicos permite focalizar el objeto de interés de los usuarios del mismo, frente a la dispersión de temas presente en un directorio web.

Todo usuario autor o editor de iSGAT podrá proponer la inserción de nuevos documentos y términos de indización en el repositorio, por lo que su actualidad y crecimiento dependerán directamente del interés del grupo de trabajo. En cierto modo, iSGAT es un sistema democrático que delega la calidad y efectividad del sistema global en la responsabilidad de sus usuarios de manera similar a la confianza que el motor de búsqueda Google delega en los creadores de páginas web a la hora de insertar enlaces.

\section{Estructura relacional de iSGAT}

El sistema iSGAT se va a desarrollar sobre un entorno de programación PHP y MySQL, de software libre y que estará abierto a posibles modificaciones por parte de todos los usuarios interesados en el mismo.

Las razones del empleo de esta plataforma son lógicas, pues este sistema nace con una voluntad de facilitar la integración de los esfuerzos de distintos equipos de trabajo a través de la web. Estos equipos precisan de una plataforma única para el desarrollo de sus trabajos que esté libre de los problemas relacionados con el copyright o la propiedad industrial de la aplicación informática. Tanto el lenguaje de programación PHP como el gestor de bases de datos relacional MySQL lo permiten; y, además, son herramientas informáticas lo suficientemente poderosas como para desarrollar iSGAT con todas las garantías de éxito.

El diseño de tesauros sobre un esquema de base de datos relacional ha sido suficientemente debatido por nuestro grupo de investigación (Rodríguez Muñoz et al., 1990; Martínez Méndez et al., 1992; Pastor Sánchez, 1992; Rodríguez Muñoz, 1992; Rodríguez Muñoz et al., 1995), quedando patente el alto grado de implicación entre un lenguaje documental claramente asociativo y un gestor de

Scire. $9: 2$ (jul.-dic. 2003) 85-98. 
bases de datos basado en las relaciones entre entidades. La posibilidad de integrar tesauros diseñados sobre entornos relacionales en hipertextos también ha sido adelantada por nuestro grupo de investigación (Pastor Sánchez y Saorín Pérez, 1995; 1998). Así pues, iSGAT es la plasmación de una serie de iniciativas y postulados teóricos anteriormente realizados dentro de una línea común de trabajo.

Una vez implementado, los distintos usuarios de iSGAT confluirán en un portal en el cual, dependiendo de su perfil, podrán realizar las tareas que tengan asignadas. Cada usuario o grupo de usuarios asociados a un tesauro específico dispondrán de una cuenta, implícitamente asociada a una serie de tablas en el gestor MySQL vinculadas a este tesauro. Las tablas son las siguientes:

- Usuarios: Almacena los datos de identificación de los usuarios del tesauro, el "login" y la palabra de acceso, y sus funciones asignadas. También refleja si el usuario está o no activo.

- Perfiles: Define los distintos perfiles de usuario del sistema y el conjunto de funciones asignadas a cada perfil.

- Fuentes: Recoge la fuente utilizada para la edición de los contenidos del tesauro, qué autor la ha empleado, el año de edición de la fuente y la fecha de la última consulta (muy importante si se trata de una página web).

- Términos: Recoge todos los términos que forman parte del tesauro, su nota explicativa, qué usuario tipo autor lo ha incorporado y la fecha de la última modificación, que en primera instancia coincide con la de creación.

- Referencias: Vincula cada término con sus fuentes.

- Relaciones: Recoge los términos relacionados y el indicador del tipo de relación existente entre ellos.

- Documentos: Si iSGAT es usado como repositorio de conocimiento, en esta tabla se almacenan las referencias a las páginas web que forman parte del mismo, junto con la fecha de su última actualización.

- Indización: Vincula a los documentos del repositorio con los términos de indización, en función del análisis de contenido realizado.

Sus campos se describn en las tablas II a IX.

\section{Conclusiones}

Con el desarrollo de un sistema de esta naturaleza y envergadura creemos que se aporta una herramienta muy eficaz para la coordinación de usuarios en la elaboración de tesauros cooperativos a través de la web o de una intranet.

En el caso de la web, se está ofreciendo una alternativa muy interesante a la organización por categorías de los directorios de recursos, que podría ser per-

Scire. $9: 2$ (jul.-dic. 2003) 85-98. 


\begin{tabular}{|l|l|}
\hline id_usuario & Identificador del usuario \\
\hline Nombre & Nombre del usuario \\
\hline Login & $\begin{array}{l}\text { Identificación de entrada al sistema } \\
\text { (junto con el contenido del campo } \\
\text { "clave") }\end{array}$ \\
\hline Clave & $\begin{array}{l}\text { Palabra clave de entrada al sistema } \\
\text { (junto con el contenido del campo } \\
\text { "login") }\end{array}$ \\
\hline Email & Correo electrónico del usuario \\
\hline id_perfil & Clave ajena de la tabla Perfiles \\
\hline patronusuario & $\begin{array}{l}\text { Descripción del conjunto de funciones } \\
\text { asignadas mediante patrón binario. }\end{array}$ \\
\hline Estado & Activo / No Activo \\
\hline
\end{tabular}

Tabla II. Descripción de la tabla Usuarios.

\begin{tabular}{|l|l|}
\hline id_perfil & Identificador del perfil de usuario \\
\hline patronperfil & $\begin{array}{l}\text { Descripción de las funciones asignadas } \\
\text { mediante patrón binario. }\end{array}$ \\
\hline
\end{tabular}

Tabla III. Descripción de la tabla Perfiles.

\begin{tabular}{|l|l|}
\hline id_fuente & Titulo \\
\hline Autores & Clave ajena de la tabla Usuarios \\
\hline Año & Año de la edición de la fuente \\
\hline Fecha & Fecha de la última consulta \\
\hline
\end{tabular}

Tabla IV. Descripción de la tabla Fuentes.

\begin{tabular}{|l|l|}
\hline id_termino & descripción \\
\hline Tipo & Nota \\
\hline Fecha_creación & Fecha de modificación \\
\hline id_pusuario & id_vusuario \\
\hline
\end{tabular}

Tabla V. Descripción de la tabla Términos

\begin{tabular}{|l|}
\hline id_termino1 \\
\hline id_termino2 \\
\hline Tiporelacion \\
\hline id_pusuario \\
\hline id_vusuario \\
\hline
\end{tabular}

\begin{tabular}{|l|}
\hline id_termino \\
\hline id_fuente \\
\hline Localización \\
\hline
\end{tabular}

\begin{tabular}{|l|}
\hline id_documento \\
\hline Titulo \\
\hline url \\
\hline Fecha_actualizacion \\
\hline
\end{tabular}

\begin{tabular}{|l|}
\hline id_termino \\
\hline id_documento \\
\hline
\end{tabular}

Tablas VI-IX. Descripción de las tablas Referencias, Relaciones, Documentos e Indización (de izquierda a derecha).

fectamente integrable en estos sistemas mediante la realización de los cambios oportunos (aunque implicaría costosos procesos de re-indización, por lo cual quedaría restringido su alcance a nuevos proyectos).

Finalmente, la gestión conjunta y en colaboración de tesauros, fuentes y recursos de información, conforman un entorno de gestión del conocimiento muy apropiado para organizaciones o comunidades virtuales de usuarios; por no hablar de las amplias posibilidades que se presentan en el campo de la difusión de la información y documentación administrativa (López Carreño et al, 1999). 


\section{Referencias}

De la Cueva Martín, A. (2000) Acceso y utilización de tesauros en Internet [En línea]. Barcelona: Universidad, 2000. URL: <http://bd.ub.es/pub/fburg/docs/cueva.pdf>. Consulta: 29 de noviembre de 2003.

López Carreño, R.; Martínez Méndez, F. J., Saorín Pérez, T.; García Gómez, J. C. (1999). El Tesauro como herramienta en la optimación de la gestión de la documentación administrativa. [En línea]. URL: <http://www.um. s/gtiweb/fjmm/isko99.html>. Consulta: 21 de noviembre de 2003.

Martínez Méndez, F. J.; Rodríguez Muñoz, J. V.; Martínez Méndez, L. (1992). Diseño lógico conceptual de tesauros. // Actas de las 4es Jornades Catalanes de Documentació. Barcelona: SOCADI, 1992.

Martínez Méndez, F. J.; García Gómez, J. C. (2003). Tesauro de redes de ordenadores. Murcia: Universidad, 2003. URL: <http://www.um.es/gtiweb/fjmm/tesauro>. [Consulta: 10 de noviembre de 2003]. Recibe más de mil visitas al mes.

Méndez Rodríguez, E. (2002a). Soporte a la construcción de Tesauros en Internet [En línea]. Getafe: Universidad Carlos III, 2002. URL: <http://rayuela.uc3m.es/ mendez/ tesauro.htm>. Consulta: 21 de diciembre de 2003.

Méndez Rodríguez, E. (2002b). Construcción de Tesauros. Madrid: Universidad Carlos III, s. d. URL: <http://rayuela.uc3m.es/ mendez/tesauro.htm> [Consulta: 20 de octubre 2003].

Moya Martínez, G. y Gil Leiva, I. (2001) Evaluación de software de gestión de tesauros. // Ciencias de la Información. 32:3 (2001) 3-23.

Pastor Sánchez, J. A. (1992) Diseño, desarrollo implementación de un sistema gestor de automatización de tesauros [Proyecto Fin de Carrera]. Murcia: Universidad, 1992.

Pastor Sánchez, J. A.; Saorín Pérez, T. (1996). El Tesauro en Hipertexto. // Scire. 2:1 (1996).

Pastor Sánchez J. A. y Saorín Pérez, T. (1995). El hipertexto documental como solución a la crisis conceptual del hipertexto: El reto de los documentos cooperativos en redes. [En línea]. // Cuadernos de Documentación Multimedia. 4 (1995).

Rodríguez Muñoz, J. V.; Martínez Méndez, F. J.; Díaz Ortuño, P. (1990). Los modelos de datos como alternativa en la construcción de tesauros. // Actas de las III Jornadas Españolas de Documentación Automatizada. Palma de Mallorca: FESABID, 1990.

Rodríguez Muñoz, J. V. (1992). Construcción del esquema conceptual del tesauro mediante un modelo de datos [Tesis Doctoral-Microficha]. Murcia: Universidad, 1992.

Rodríguez Muñoz, J. V.; Díaz Ortuño, P. M.; Martínez Méndez, F. J.; Moya Martínez, G. (1995). Reglas de producción para una base de conocimientos en la construcción de tesauros. // Álvarez Osorio, J. (ed). New Worlds in information and documentation: Proceedings of the Forty-Sixth FID Conference and Congress. Amsterdam, The Netherlands: Elsevier, 1994. 341-352. 